\title{
The Estimation of the Stability of Plants Constituting the Photosynthesizing Unit of Bioregenerative Life Support Systems for Including Them into the Plant Waste Mass Exchange
}

\author{
Sofiya A. Ushakova*, Alexander A. Tikhomirov, Vladimir V. Velichko, \\ Igor G. Zolotukhin, Yuriy A. Kudenko and Tamara K. Golovko \\ Institute of Biophysics of Siberian Branch of Russian Academy of Sciences, \\ Akademgorodok, Krasnoyarsk, 660036 Russia \\ Institute of Biology of Komi Scientific Centre of Urals Branch \\ of Russian Academy of Sciences, \\ 28 Kommunisticheskaya str., Syktyvkar, 167610 Komi Republic, Russia ${ }^{1}$
}

Received 1.09.2007, received in revised form 1.12.2007, accepted 15.01.2008

One of the ways of increasing the closure of mass exchange processes in the bioregenerative life support systems (BLSS) may be the technology of plant cultivation on the so-called soil-like substrate (SLS) which is expected to utilize inedible plant biomass with the help of the SLS heterotrophic link. Earlier, based on the example of the radish inedible biomass, there was shown the principal possibility of its successful biological combustion (without decreasing the productivity of the plants cultivated) during their direct introduction into the SLS. The possibility of a direct plant waste introduction into the SLS could considerably simplify the technological processes of their biological combustion and increase their efficiency. However, there were no experiments carried out with respect to the other vegetables included into the BLSS photosynthesizing link. The aim of the given work is estimating the possible influence of the inedible biomass of different plant species introduced into the SLS, on the plant productivity. Several series of the test experiments were carried out. In the first experiment series, dried radish leafy tops making up $0.8 \mathrm{~kg} / \mathrm{m}^{2}$ of the sown area, dried carrot leafy tops $-3.1 \mathrm{~kg} / \mathrm{m}^{2}$ and dried beet leafy tops $-2.5 \mathrm{~kg} / \mathrm{m}^{2}$ were introduced into the SLS makeup. As a test culture, there was taken the radish Raphanus sativus L of the Virovsky Beliy species. In the second experiment series, before the sowing of radish and wheat, there were introduced $1.2 \mathrm{~kg} / \mathrm{m}^{2}$ and $3.1 \mathrm{~kg} / \mathrm{m}^{2}$ of dry wheat straw into the SLS respectively. In the third series of the experiments, the wheat straw, before being introduced into the SLS, was subjected to the preliminary processing. In the fourth experiment series, there was studied the supposition about the possibility of decreasing the negative influence of introducing the vegetable tops into the SLS on the plant growth of the succeeding generation under the conditions of growing plants on a various-ages multi-species vegetable conveyor. According to the results of the research, the direct introduction of carrot and beet tops, as well as dry wheat straw, resulted in the considerable drop of the plant productivity. The vegetable cultivation in a conveyor mode done for three generations, also resulted in their productivity decrease. It was shown that the straw mineralization by using the method of "wet incineration" developed by $Y u$. A. Kudenko had completely removed the negative effect of the plant waste on the plant growth and could be the alternative way of the wheat waste utilization.

Keywords: wheat Triticum aestivum L., radish Raphanus sativus L., carrot Daucus carota L., beet Beta vulgaris L., productivity, mineral composition, soil-like substrate.

\footnotetext{
Corresponding author E-mail address: ubflab@ibp.ru

(C) Siberian Federal University. All rights reserved
} 


\section{Introduction}

One of the main problems of creating bioregenerative life support systems (BLSS) working for a long time, is connected with the necessity of increasing the closure of mass exchange. This would allow decreasing the reserves of initial substances and the amount of the waste being stored. And the life quality of the crew must be high enough. One of the ways of increasing the closure of mass exchange is the involving into the rotation the substances of inedible biomass of the plants constituting the BLSS phototrophic link. For this purpose, N.S. Manukovsky et al. (1997) suggested the technology of the plant cultivation on the so-called soil-like substrate (SLS which is supposed to utilize the inedible plant biomass with the help of the SLS heterotrophic link. In our opinion, the two principles of forming the BLSS phototrophic link, in which the substrate for the plant cultivation may be a SLS, are of interest.

The first principle: the growing of all the plants forming the composition of the vegetable link of BLSS is carried out on the SLS. All the inedible plant biomass must be returned to the SLS. Instead of the edible biomass, to make up for the mineral elements carried out with it, it is supposed to carry in a certain amount of mineralized and specially prepared solid and liquid excreta of a man. In this case, the oxygen educed during the photosynthetic processes when synthesizing the inedible plant biomass, almost all of it, will be used for oxidizing this biomass with the SLS heterotrophic link. During the experiments on the experimental modeling of artificial ecological systems, we showed that the productivity of wheat and radish cultivated in a conveyor mode on the SLS, was not lower than the productivity of these cultures, cultivated in a conveyor mode on the neutral substrate in the complex BIOS (Tikhomirov et al., 2003 a, b).

The second principle consists in cultivating wheat, the main cereal crop making up the composition of the system vegetable link, on the neutral substrate. Into the latter, a certain amount of mineralized and specially prepared solid and liquid excreta is introduced. It also includes cultivating vegetable plants on the SLS. In this case, the inedible vegetable biomass will be returned to the SLS. Instead of the edible biomass, to make up for the mineral elements carried out with it, it is supposed to bring in to the substrate a certain amount of the straw which also will be utilized by the SLS heterotrophic link. The part of the straw which is not included into the mass exchange will have to be stored. Following from the supposed link composition of higher plants (Gitelson et al., 2003), the part of vegetables in the diet must make up not more than $30 \%$ of the daily diet of a man. So the oxygen consumption for the decomposition of the inedible part of vegetable plants and straw introduced into the SLS instead of the edible part of the vegetables will be considerably less as compared to cultivating plants according to the first principle.

When forming the phototrophic unit according to the first principle as well as when forming it according to the second one, one of the main problems is the way of introducing the plant waste into the substrate. In the abovequoted works (Tikhomirov et al., 2003 a, b) before introducing straw into the SLS, it was subjected to a number of successive processing operations (that is pasteurization, fermentation, growing fungi). Such a multi-stage preparation of the straw before introducing it into the substrate considerably complicates the process of preparing the substrate using straw for plant cultivation. But in these experiments, just after drying, the inedible radish biomass was introduced into the substrate which preceded sowing the radish seeds. During this process, no disturbances of the radish growth were found, when it was grown on such a substrate. But the fact that the introduction of the inedible radish biomass has no effect on the 
plant growth processes does not mean that such an introduction of the inedible biomass of other vegetable cultures will not result in worsening the processes of the plant growth and development. Besides, by analogy with introducing the inedible radish biomass, the simplest solution of the problem of introducing straw would be its introduction into the SLS just after the gathering of crops. So the aim of the given work is to study the reactions of the plants cultivated on the SLS, to the introduction of the inedible biomass of plants which are supposed to be the representatives of the BLSS photosynthesizing link, into the root habitat.

\section{Materials and methods}

In order to solve this task in the test mode, there was conducted a series of experiments with the utilization of the newly - prepared SLS according to the method developed by N. S. Manukovsky et al. (1997). Based on the structure of the BLSS vegetable link, which was tested in the multi-day experiment carried out at the Institute of Biophysics in the Siberian Branch of the Russian Academy of Sciences (IBP SB RAS) (Lisovsky and Shilenko, 1975; Gitelson et al., 2003), as the vegetable waste, we used the preliminarily dried wheat straw and the inedible biomass of radish, carrot and beet. As the test cultures, there was taken the radish Raphanus sativus L, as the representative of vegetable cultures, and the spring wheat (Triticum aestivum L, line 232 of the selection of G. M. Lisovsky) as the main culture for forming the vegetable diet of the European type. The duration of cultivating the radish was 30 days, and that of the wheat -60 days.

The plants were grown in the vegetation chamber on the condition of the natural concentration of $\mathrm{CO}_{2}$, the twenty-four-hour lighting and at the air temperature of $24{ }^{\circ} \mathrm{C}$. The planting density for the wheat made up 1000 plants per $1 \mathrm{~m}^{2}$ of the planting area, for the radish it was 190 plants per $1 \mathrm{~m}^{2}$ of the planting area. For lighting there were used the lamps DKsTV -6000 , the headlight intensity was $150 \mathrm{~W} / \mathrm{m}^{2}$. The watering of the plants was carried out by the irrigation solution $50 \mathrm{l} / \mathrm{m}^{2}$ of the planting. The irrigation solution contained the settled running water having the extractive substances of the SLS dissolved in this water. During the plant growth, in the irrigation solutions there was measured $\mathrm{pH}$ and analyzed the content of different forms of nitrogen. The method of introducing the vegetable waste in more detail will be given when describing the experiment results.

Besides, there was conducted the estimation of the influence of introducing the inedible vegetable biomass into the SLS on the growth of the next generation of these cultures for several generations of the vegetables. For this purpose, a three-type vegetable conveyor of different ages was formed. The plants contained 3 ages of the carrot (Daucus carota L) of the sort "Vitaminnay 6". They also contained the beet (Beta vulgaris L) of the sort "Yegipetskaya", and 2 ages of the radish (Raphanus sativus L) of the sort "Virovsky beliy". The duration of the vegetation of one generation of the carrot and beet made up 78 days, for the radish it was 26 days. After each harvest before the next planting, the inedible biomass was introduced into the substrate to cultivate the plant of the same kind. Instead of the edible biomass, into the irrigation solution, there was gradually introduced the wheat straw mineralized with hydrogen peroxide at the temperature $90{ }^{\circ} \mathrm{C}$ in the alternating electromagnetic field according to the method developed by Yu. A. Kudenko et al. (1997, 2000). During the vegetation, the mineralized solution was evenly added into the irrigation solution for watering the plants. The amount of the introduced mineralized solution was calculated following from the nitrogen content in the edible plant biomass. The irrigation solution 
used for watering the plants was the same for all the plants constituting the conveyor composition. The density of planting the carrot was 150 plants per $1 \mathrm{~m}^{2}$, the density of planting the beet was 100 ones per $1 \mathrm{~m}^{2}$, for the radish it was 250 plants per $1 \mathrm{~m}^{2}$. The rest of the conditions for the cultivation were similar to those of growing the plants in the above - described experiments.

\section{Results and discussion}

1. The influence of introducing

the inedible biomass of the radish,

carrot and beet into the SLS

Based on the numerous experiments on growing plants on the conditions of the intensive light culture carried out at the IBP SB RAS, as a basis of calculating the amount of the inedible biomass, we took, the plant productivity of radish, carrot and beet when cultivated on the neutral substrate on the optimum environment conditions (Table 1) (Lisovsky and Shilenko, 1979). Following from the mass of the inedible tops of the vegetables (Table 1), depending on the experiment chosen, before sowing the plants, into the SLS there was brought in the dry tops of the radish mass $-0.8 \mathrm{~kg} / \mathrm{m}^{2}$ of the planting (treatment 1). There was also brought in the dry tops the carrot mass $-3.1 \mathrm{~kg} / \mathrm{m}^{2}$ (treatment 2), or the dry tops of the beet mass $-2.5 \mathrm{~kg} / \mathrm{m}^{2}$ (treatment 3 ). The total number of the macronutrient elements contained in the SLS and introduced in to the substrate with the corresponding inedible biomass, is shown in Table 2.

Into the substrate prepared in the above described way, the seeds of the radish Raphanus sativus L of the sort "Virovsky beliy" were sown. The plants having the age of 30 days reached the state of a technical ripeness. Then they were gathered in. And the comparative estimation of the harvest yield was carried out.

The periodic measurement, during the plant growth, of $\mathrm{pH}$ of the irrigation solutions, showed that in all the treatments, the irrigation solution had a low-alkaline reaction. The lowest $\mathrm{pH}$, equal to about 8 , was with the solution for watering the radish of treatment 1 , and the highest $(8.6-9)$ was for the solution for watering the radish of treatment 3 (Table 3 ).

Table 1. The Average Harvest of the Plants Grown on the Neutral Substrate on the Optimum Environment Conditions (Lisovsky and Shilenko, 1975)

\begin{tabular}{|l|r|r|r|r|r|r|}
\hline \multirow{2}{*}{$\begin{array}{c}\text { The analyzed part } \\
\text { of the plant }\end{array}$} & \multicolumn{2}{|c|}{ Radish, $\mathrm{kg} / \mathrm{m}^{2}$} & \multicolumn{2}{c|}{ Carrot, $\mathrm{kg} / \mathrm{m}^{2}$} & \multicolumn{2}{c|}{ Beet, $\mathrm{kg} / \mathrm{m}^{2}$} \\
\cline { 2 - 8 } & \multicolumn{1}{c|}{ wet } & \multicolumn{1}{c|}{ dry } & \multicolumn{1}{c|}{ wet } & \multicolumn{1}{c|}{ dry } & wet & \multicolumn{1}{c|}{ dry } \\
\hline Tops & 11.5 & 0.8 & 11,7 & 3,1 & 18,6 & 2,5 \\
\hline Root-crops & 10.1 & 0.8 & 24,2 & 4,4 & 35,5 & 3,8 \\
\hline Roots & 0.8 & 0.1 & 6,0 & 0,4 & 1,9 & 0,3 \\
\hline Total & 22.4 & 1.7 & 41,9 & 7,9 & 56,0 & 6,6 \\
\hline
\end{tabular}

Table 2. The quantity of the mineral elements introduced into the SLS with the inedible biomass and which are present in the initial substrate, $\mathrm{g} / \mathrm{m}^{2}$

\begin{tabular}{|l|c|r|r|r|r|r|r|r|}
\hline $\begin{array}{c}\text { The plant tops } \\
\text { biomass }\end{array}$ & $\begin{array}{c}\text { Introduced dry } \\
\text { waste, } \mathrm{kg} / \mathrm{m}^{2}\end{array}$ & $\mathrm{Na}$ & $\mathrm{K}$ & $\mathrm{Ca}$ & $\mathrm{Mg}$ & $\mathrm{P}$ & $\mathrm{S}$ & $\mathrm{N}_{\text {total }}$ \\
\hline Radish & 0,8 & 1,6 & 34,4 & 29,7 & 4,7 & 1,6 & 4,7 & 26,6 \\
\hline Carrot & 3,1 & 28,1 & 75,0 & 59,4 & 15,6 & 6,3 & 12,5 & 98,0 \\
\hline Beet & 2,5 & 40,0 & 105,0 & 30,0 & 30,0 & 7,5 & 12,5 & 74,3 \\
\hline $\begin{array}{l}\text { The initial SLS, } \\
20,3 \mathrm{~kg} / \mathrm{m}^{2}\end{array}$ & & 20,3 & 182,8 & 589,1 & 121,9 & 60,9 & 101,6 & 595,2 \\
\hline
\end{tabular}


Table 3. The contents of different forms of nitrogen and $\mathrm{pH}$ of the irrigation solutions depending on the type of the vegetable waste introduced into the SLS (the error did not exceed $10 \%$ of the measured value)

\begin{tabular}{|c|c|c|c|c|c|}
\hline \multirow{2}{*}{$\begin{array}{l}\text { The plant age, } \\
\text { twenty-four hours } \\
\text { after the shoots }\end{array}$} & \multirow{2}{*}{$\begin{array}{c}\text { The vegetable } \\
\text { waste }\end{array}$} & \multicolumn{3}{|c|}{ Nitrogen forms, $\mathrm{mg} / \mathrm{l}$} & \multirow{2}{*}{$\begin{array}{l}\mathrm{pH} \text { of the } \\
\text { solution }\end{array}$} \\
\hline & & nitrate & ammonia & reduced & \\
\hline \multirow{3}{*}{10} & Radish & 164,2 & 1,9 & 39,0 & 8,1 \\
\hline & Carrot & 7,1 & 5,7 & 63,7 & 8,6 \\
\hline & Beet & 15,0 & 5,3 & 46,0 & 8,6 \\
\hline \multirow{3}{*}{16} & Radish & 135,4 & 1,4 & 28,7 & 8,3 \\
\hline & Carrot & 11,2 & 3,4 & 42,3 & 8,6 \\
\hline & Beet & 13,8 & 1,9 & 49,3 & 9,0 \\
\hline \multirow{3}{*}{19} & Radish & 116,3 & 2,1 & 47,3 & 8,2 \\
\hline & Carrot & 16,0 & 3,7 & 62,7 & 8,6 \\
\hline & Beet & 23,3 & 3,9 & 76,0 & 8,8 \\
\hline \multirow{3}{*}{24} & Radish & 49,9 & 1,0 & 23,3 & 7,9 \\
\hline & Carrot & 12,0 & 1,9 & 42,0 & 8,5 \\
\hline & Beet & 10,0 & 2,1 & 48,0 & 8,7 \\
\hline \multirow{3}{*}{30} & Radish & 8,0 & 0,8 & 15,3 & 7,9 \\
\hline & Carrot & 8,6 & 0,9 & 28,7 & 8,5 \\
\hline & Beet & 10,5 & 2,4 & 67,7 & 8,8 \\
\hline
\end{tabular}

The introduction of the $3.1 \mathrm{~kg} / \mathrm{m}^{2}$ carrot tops and the $2.5 \mathrm{~kg} / \mathrm{m}^{2}$ beet tops into the SLS, led 10 days after the vegetation of the radish started, to a sharp decrease in the content of nitrate forms of nitrogen in the irrigation solutions (as compared to the introduction of $0,8 \mathrm{~kg} / \mathrm{m}^{2}$ radish tops). Only by the moment of harvesting the plants, the content of the nitrate forms of nitrogen almost became even. And during the whole period of the plant growth, the content of the reduced forms of nitrogen in the irrigation solutions of treatments 2 and 3 , was higher than in the solution of treatment 1 .

The comparative estimation of the radish harvest structure showed that the wet and dry tops of treatment 1 turned out to be 2.6 and 3 times higher respectively than for plants of treatment 2 and 4.1 and 3.7 times higher respectively than for plants of treatment 3 (Table 4). The tops plant biomass of treatment 3 appeared to be a little less than the tops of treatment 2. Approximately the same ratio is found when estimating the roots mass of the studied treatments. The wet and dry roots mass of treatment 1 was 2.6 and 3.1 times higher respectively than that for the plants of treatments 2 and 3. As a result, the total plant mass of the plants of treatment 1 was considerably higher than the total plant mass of the plants of treatment 2 and 3 .

It should be noted that despite the differences in the plant biomass, the part of the economically useful biomass in all the biomass actually did not depend on the plant waste introduced into the SLS (Table 4).

So, the introduction of the inedible plant biomass of carrots and beet in quantities approximately equal to their harvest when cultivating them on the same area, leads to a considerable drop of the plant productivity. The harvest of the radish, grown on the SLS with the addition of the radish tops did not actually differ from the radish harvest grown on the neutral substrate on the similar environmental conditions. There may by various reasons for the negative influence of introducing the carrot and beet tops into the SLS. 
Table 4. The radish tops and root-crops depending on the kind of the vegetable waste introduced into the SLS

\begin{tabular}{|l|c|c|c|c|c|}
\hline \multirow{2}{*}{ Treatments } & \multicolumn{2}{|c|}{$\begin{array}{c}\text { The tops mass, } \\
\mathrm{kg} / \mathrm{m}^{2}\end{array}$} & \multicolumn{2}{c|}{$\begin{array}{c}\text { The root-crops mass, } \\
\mathrm{kg} / \mathrm{m}^{2}\end{array}$} & \multirow{2}{*}{ Harvest index } \\
\cline { 2 - 5 } & wet & dry & wet & dry & \\
\hline Treatment $1 *$ & $5,8 \pm 1,8$ & $0,75 \pm 0,23$ & $8,5 \pm 2,7$ & $0,68 \pm 0,17$ & 0,48 \\
\hline Treatment $2 * *$ & $2,2 \pm 0,4$ & $0,25 \pm 0,04$ & $3,3 \pm 0,7$ & $0,23 \pm 0,05$ & 0,48 \\
\hline Treatment $3 * *$ & $1,4 \pm 0,3$ & $0,20 \pm 0,05$ & $2,7 \pm 0,6$ & $0,21 \pm 0,05$ & 0,51 \\
\hline
\end{tabular}

* $0.8 \mathrm{~kg}$ of the dry radish tops is added into the SLS

** $3.1 \mathrm{~kg}$ of the dry carrot tops is added into the SLS

$* * * 2.5 \mathrm{~kg}$ of the dry beet tops is added into the SLS

But the main reason, in our opinion, is the following: the introduction of $2.5-3.1 \mathrm{~kg} / \mathrm{m}^{2}$ dry organic substance could lead to the activation of the growth processes of microorganisms which must result in the decrease in the content of the nitrogen forms accessible for the plants (Table 3). The speed of releasing the nitrogen combined in the organic substance of the vegetable waste did not compensate the speed of combining the nitrogen with the microorganisms. The shortage of the accessible nitrogen forms may be one of the main reasons for dropping the plant productivity.

At the same time, one cannot exclude the fact that in the inedible plant biomass, there may be the substances leading to the retardation of the plant growth processes. When a considerable amount of the plant waste is introduced into the SLS, the contents of these substances may become sufficient to exert a negative influence on the plant growth and development processes.

2. The influence of introducing straw into the SLS on the wheat and radish growth and development

In case of using the SLS for the wheat cultivation, the inedible wheat plant biomass for reducing the content of the mineral elements used for its formation in the substrate, also has to be returned into the SLS. Therefore, we carried out the estimation of the possible influence of introducing the dry straw on the wheat growth and development. For this purpose, there was taken the newly - prepared SLS which was divided into 2 parts. One of the parts was used for growing the wheat without introducing the straw. Into the second part, there was added dry straw in the quantity of $3.1 \mathrm{~kg} / \mathrm{m}^{2}$ of the planting. The amount of the introduced straw was taken as following from the preliminary experiments on cultivating wheat on the newly - prepared SLS, in which the harvest reached $2.5 \mathrm{~kg} / \mathrm{m}^{2}$ for the cereals, and $3.1 \mathrm{~kg} / \mathrm{m}^{2}$ for the straw. The settled running water was taken as a basis for watering the plants. The plants were watered once a day. Thus, the irrigation solution contained the running water and the extractive compounds from the SLS. The rest of the conditions for conducting the experiments are similar to those described in the previous part of the work.

The comparative estimation of the structure of the harvest of the wheat grown on the SLS and on the SLS with the addition of straw is given in Table 5. The introduction of straw into the substrate led to a considerable retardation of the plant growth and development processes. The height of the plants and the length of the stem decreased with respect to the control almost by $40 \%$. The bushing out decreased by $40 \%$. And the number of productive sprouts decreased by $18 \%$. The structure of the ear itself also changed. As a result, the total number of the ears in the ear of the main sprout decreased. And the number of the productive ears decreased by $40 \%$. 
Table 5. The harvest structure of the wheat grown on the SLS and on the SLS with the addition of straw

\begin{tabular}{|l|c|c|c|c|c|c|}
\hline \multirow{2}{*}{ Treatment } & \multicolumn{2}{|c|}{ The height, $\mathrm{cm}$} & \multicolumn{2}{c|}{$\begin{array}{c}\text { The number of the } \\
\text { sprouts } / \mathrm{m}^{2}\end{array}$} & \multicolumn{2}{c|}{$\begin{array}{c}\text { The number of the } \\
\text { spikelets in the ear }\end{array}$} \\
\cline { 2 - 7 } & of the plant & of the stem & total & $\begin{array}{c}\text { the } \\
\text { productive } \\
\text { ones }\end{array}$ & total & productive \\
\hline $\begin{array}{l}\text { The SLS } \\
\text { control }\end{array}$ & $50,7 \pm 1,2$ & $44,7 \pm 1,2$ & $2500 \pm 269$ & $1100 \pm 100$ & $12 \pm 0,2$ & $10 \pm 0,3$ \\
\hline $\begin{array}{l}\text { The SLS } \\
\text { straw }\end{array}$ & $32,0 \pm 0,6$ & $27,1 \pm 0,6$ & $1500 \pm 224$ & $900 \pm 100$ & $9 \pm 1$ & $6 \pm 0,8$ \\
\hline
\end{tabular}

The retardation of the plant growth processes influenced the wheat harvest (Fig. 1). The total harvest and the harvest of the wheat cereals decreased almost by $60 \%$ as compared to the control. And the mass of 1000 grains appeared to be $16 \%$ smaller than the mass of 1000 grains of the control plants.

In case of organizing a vegetable conveyor in the system according to the second principle (see Introduction), to grow vegetables for the purpose of making up for the carrying out of the elements with the edible biomass, it is supposed to introduce straw. So, when calculating the quantity of the dry straw necessary for introducing into the SLS for growing radish, we proceeded from the assumed roots mass which may be obtained when growing radish on it and from comparing the mineral composition of the radish and straw roots grown on the neutral substrate (Lisovsky and Shilenko, 1975).

It can be seen from Table 6 , that the main difference between the mineral composition of the straw and the roots is connected with the content of nitrogen and sulphur, the relative quantity of which in the roots is 1.5 times higher than in the straw. When the roots harvest is $0.8 \mathrm{~kg} / \mathrm{m}^{2}$, to make up for the nitrogen carried out with the roots, it is necessary to introduce the straw into the SLS the mass of which is approximately 1.5 times more. So, when carrying out the experiments, we introduced

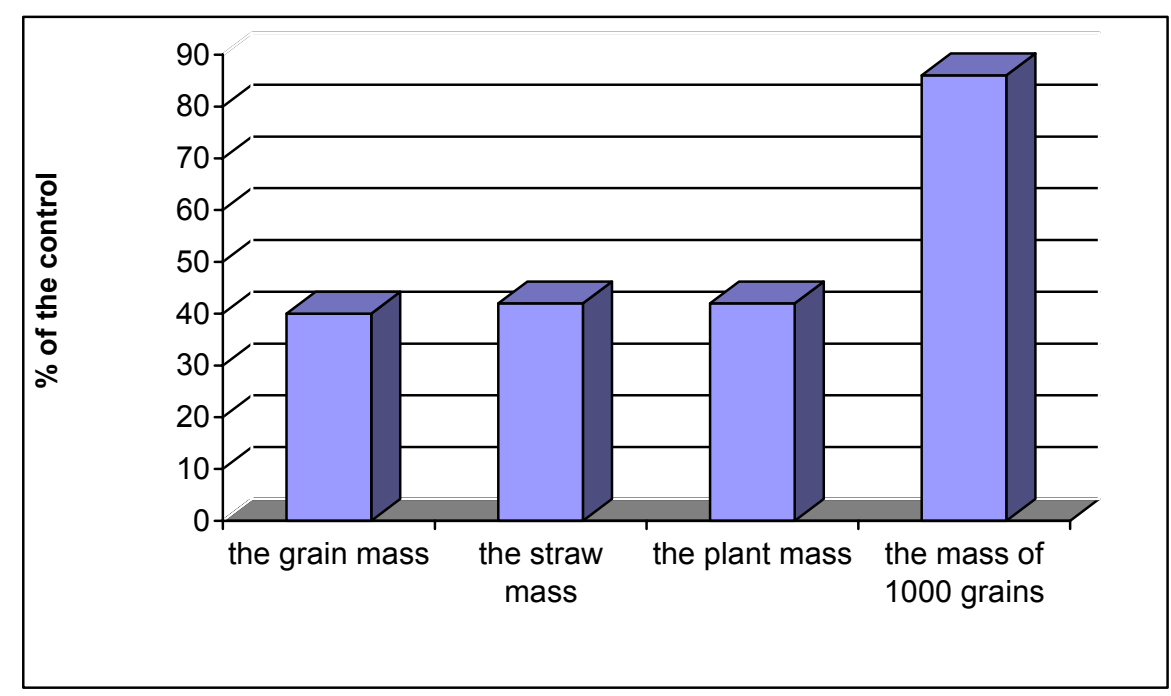

Fig. 1. The harvest of the wheat grown on the SLS with the addition of straw, \% of the control 
Table 6. The mineral composition of the wheat straw, radish root-crops and the water extract from the wheat straw

\begin{tabular}{|l|c|c|c|c|c|c|}
\hline \multicolumn{1}{|c|}{ The object } & $\mathrm{P}$ & $\mathrm{K}$ & $\mathrm{Ca}$ & $\mathrm{Mg}$ & $\mathrm{S}$ & $\mathrm{N}_{\text {total }}$ \\
\hline $\begin{array}{l}\text { The wheat straw, \% } \\
\text { in the dry substance }\end{array}$ & $0,68 \pm 0,07$ & $5,22 \pm 0,51$ & $1,43 \pm 0,16$ & $0,36 \pm 0,04$ & $0,41 \pm 0,04$ & $1,8 \pm 0,2$ \\
\hline $\begin{array}{l}\text { The radish root- } \\
\text { crops, \% in the dry } \\
\text { substance }\end{array}$ & $0,73 \pm 0,08$ & $5,96 \pm 0,62$ & $0,57 \pm 0,08$ & $0,23 \pm 0,02$ & $0,77 \pm 0,08$ & $2,8 \pm 0,3$ \\
\hline $\begin{array}{l}1 \quad \mathrm{~L} \text { of the water } \\
\text { extract from } 40 \mathrm{~g} \text { of } \\
\text { the straw, } \mathrm{mg} / \mathrm{l}\end{array}$ & $115 \pm 23$ & $1150 \pm 200$ & $70 \pm 10$ & $74 \pm 12$ & $77 \pm 8$ & $135 \pm 27$ \\
\hline
\end{tabular}

Table 7. The radish harvest grown on the SLS (control) and on the SLS with the straw addition

\begin{tabular}{|l|c|c|c|c|}
\hline \multirow{2}{*}{ The part of the plant } & \multicolumn{2}{|c|}{ The control, $\mathrm{kg} / \mathrm{m} 2$} & \multicolumn{2}{c|}{ The test, $\mathrm{kg} / \mathrm{m}^{2}$} \\
\cline { 2 - 5 } & wet & dry & wet & dry \\
\hline The tops & $3,4 \pm 0,6$ & $0,47 \pm 0,10$ & $1,2 \pm 0,2$ & $0,20 \pm 0,02$ \\
\hline The root-crops & $8,9 \pm 1,8$ & $0,708 \pm 0,13$ & $1,9 \pm 0,6$ & $0,15 \pm 0,05$ \\
\hline
\end{tabular}

the straw at a rate of $1.2 \mathrm{~kg} / \mathrm{m}^{2}$ of the planting. The rest of the conditions of the cultivation are similar to those in the above described experiments.

The results of the comparative estimation of the radish harvest, grown on the SLS and on the SLS with the addition of the straw, are given in Table 7.

Cultivating radish on the SLS with the addition of straw led to decreasing the wet radish tops by 2.8 times as compared to the control. And the dry tops decreased by 2.4 times. A more considerable influence was exerted by introducing the straw upon the roots mass, of which the wet and the dry mass decreased almost by 4.7 times.

Thus, the test experiments on studying the influence of introducing dry straw into the SLS for the subsequent growing of wheat and radish on it showed that such an introduction of straw led to considerable disturbances in the plant growth and development. As a result, a considerable drop in productivity and a decrease in the economical and useful plant harvest can occur.

In the subsequent series of the experiments, the ways of preparing the wheat straw for introducing it into the substrate to clarify the possibility of decreasing the negative influence of the straw on the processes of the radish growth and development were estimated. Since by introducing straw into the SLS we suppose to compensate the mineral elements carried out with the roots, like in the previous experiment, $1.2 \mathrm{~kg} /$ $\mathrm{m}^{2}$ of dry straw was used in each treatment. The variants of the preliminary processing of the straw were as follows:

Treatment $1-1.2 \mathrm{~kg} / \mathrm{m}^{2}$ of dry straw (without processing) was introduced into the SLS;

Treatment $2-1.2 \mathrm{~kg}$ of the straw was mineralized with hydrogen peroxide at the temperature of $90{ }^{\circ} \mathrm{C}$ in the alternating electromagnetic field according to the method developed by Yu. A. Kudenko et al. (1997, 2000). The mineralized solution was evenly, during the vegetation, added into the irrigation solution for watering the radish.

Treatment 3 - the preliminarily steeped straw (at a rate of $1.2 \mathrm{~kg} / \mathrm{m}^{2}$ of the planting) was kept in the thermostat for 2 days at the temperature of $45^{\circ} \mathrm{C}$. Then the straw was squeezed out, and after that it was evenly added, during the vegetation, into the irrigation solution for watering the radish. 
The squeezed straw was introduced into the substrate before sowing the plants. The contents of the macronutrient elements in $1 \mathrm{~L}$ of the water extract made from $40 \mathrm{~g}$ of dry straw are given in Table 6. Thus, during the plant growth, 301 of the water straw extract was poured into the irrigation solution at a rate of $1 \mathrm{~m}^{2}$ of the planting.

In Fig. 2 one can see that the best way of introducing straw is its preliminary mineralization. The tops of treatment 2 were 2.5 times as large as the tops of treatment 1 and about 1. 3 times as large as the tops of treatment 3 . The plant roots mass of treatment 2 was also considerably larger than the roots mass of the rest of the treatments. The analysis of the state of the live leaves of the radish showed that the difference between the tops masses of the treatments studied is determined mainly by the difference between the live leaves masses (Table 8). Here it should be noted that the leaves water saturation of treatment 3 was somewhat higher than that of treatments 1 and 2. Since the relative leaves surface density did not differ very much among the treatments, the differences between the leaf areas of different treatments are also close to the difference between their masses.

It follows from the given results that when carrying out the test experiments, it is only the straw mineralization that removes the plant growth retardation caused by introducing the straw into

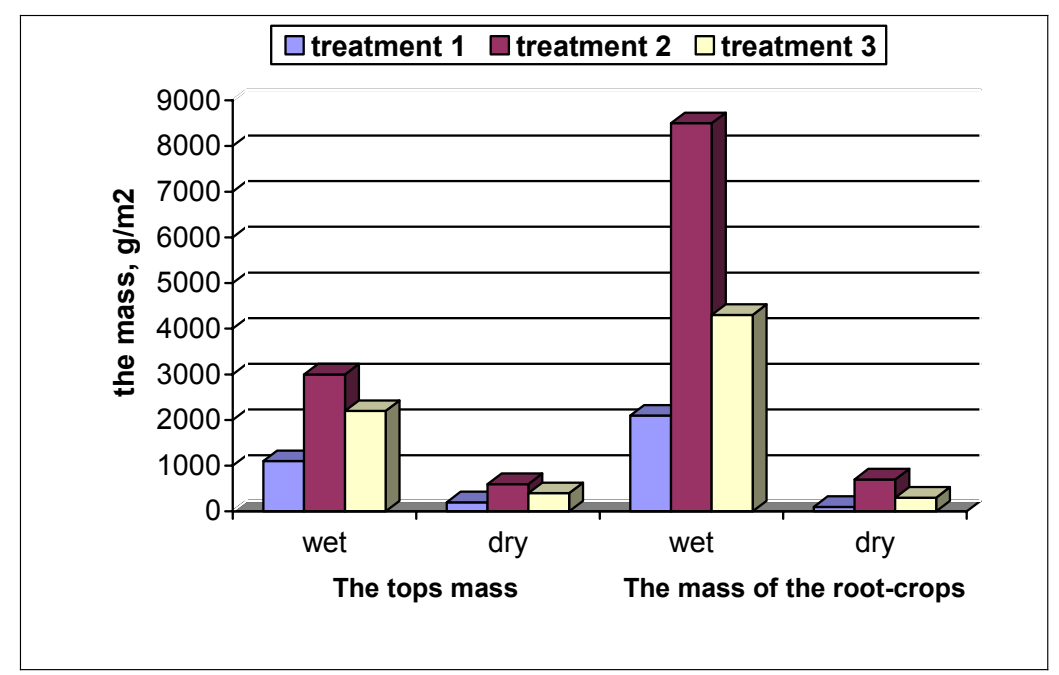

Fig. 2. The mass of the radish plant $\left(\mathrm{g} / \mathrm{m}^{2}\right)$, grown on the SLS with the addition of straw after different processing (see the description of the treatments in the text)

Table 8. The characteristics of the live radish leaves grown on the SLS with the straw addition after different processing (as calculated per 1 plant)

\begin{tabular}{|l|c|c|c|c|c|c|}
\hline \multirow{2}{*}{ Treatment * } & \multicolumn{2}{|c|}{ The leaves mass, g } & \multirow{2}{*}{$\begin{array}{c}\text { \% of the } \\
\text { dry } \\
\end{array}$} & \multicolumn{2}{|c|}{$\begin{array}{c}\text { The leaves } \\
\text { substance }\end{array}$} & \multicolumn{2}{|c|}{$\begin{array}{c}\text { The specific surface } \\
\text { leaves density, g/dm }\end{array}$} \\
\cline { 2 - 3 } & wet & dry & & wet & dry \\
\hline Treatment 1 & $670 \pm 34$ & $156 \pm 4$ & $23,3 \pm 0,7$ & $163 \pm 28$ & $4,06 \pm 0,60$ & $0,95 \pm 0,17$ \\
\hline Treatment 2 & $1762 \pm 215$ & $406 \pm 51$ & $23,0 \pm 0,1$ & $490 \pm 22$ & $3,60 \pm 0,18$ & $0,83 \pm 0,04$ \\
\hline Treatment 3 & $1298 \pm 67$ & $252 \pm 15$ & $19,4 \pm 0,1$ & $321 \pm 11$ & $4,04 \pm 0,11$ & $0,79 \pm 0,03$ \\
\hline
\end{tabular}

* - see the description of the treatments in the text 
the substrate. But the preliminary keeping of the steeped straw in the thermostat, the subsequent obtaining of the water extract and its gradual introduction into the irrigation solution during the plant growth, led to some decrease of the negative influence of introducing the straw into the SLS as compared to the treatments of introducing dry straw without any processing.

3. The influence of introducing the inedible vegetable plant biomass into the SLS on the grows of the next generation of these cultures for several generations of the vegetable conveyor

To decide the problem of introducing the plant waste, perhaps, the experiments may help on cultivating plants in the conveyor mode when using the total system of the plant watering with the irrigation solution. Under such conditions, the effect obtained from the periodic introduction of the inedible plant biomass into the substrate, will, perhaps, be smoothed over as a result of the regular exchange between the vegetation vessels connections which are in the combined irrigation solution. Thanks to this, a more or less constant order of the mineral plant feeding will be maintained. In the conveyor mode, the composition of the microflora must become stable. Besides, in the irrigation solution, the concentration of the organic substances, educed during the decomposition of the inedible biomass and capable of leading to the plant growth retardation due to the discontinuous introduction, will be considerably decreased. Therefore in the following series of the experiments, carried out in the conveyor mode, we formed a three - type biocenosis, and in the course of 3 generations, the influence of introducing the inedible vegetable plant biomass on their productivity was tested. The first plant generation was grown on the newly - prepared SLS without introducing the tops of the studied vegetable plants.
In spite of the fact that the irrigation solution was general for the vegetable cultures, the plant reactions were somewhat different (Fig. 3). The introduction of the carrot, beet and radish tops led, from one generation to another, to the gradual decrease of the dry carrot tops and did not influence the carrot roots mass. As for the beet tops, already in the second generation, they showed a considerable decrease of the roots mass. As a result of the third generation, not only the roots mass but also the leaves mass decreased. The radish tops responded the introduction of the inedible vegetable mass least of all: the leaves mass did not change from generation to generation. In the second and third generations the roots mass was smaller than in the first one. But this difference, as compared to the beet tops was not so great (Fig. 3).

It should be noted that if in the test experiments the introduction of the radish tops did not influence the radish productivity, on the conditions of using the three - type conveyor with the common irrigation solution, there was marked the decrease of the roots mass by $31 \%$. The reason for the drop in the plant productivity is evidently connected with the influence of introducing the inedible carrot and beet biomass into the SLS exerted through the irrigation solution.

\section{Conclusion}

The experiments conducted on studying the biodegradation of the inedible carrot and beet biomass, showed the drop in the radish productivity when cultivating it on the substrate with introducing the inedible biomass of these plants into the SLS in quantities, approximately equal to their harvest when cultivated on the similar conditions on the neutral substrate with using artificial mineral media. Moreover, the growing of the beet, carrot and radish tops in the conveyor mode, does not decide, as it was expected, the problem connected with the plant growth retardation, caused by introducing 

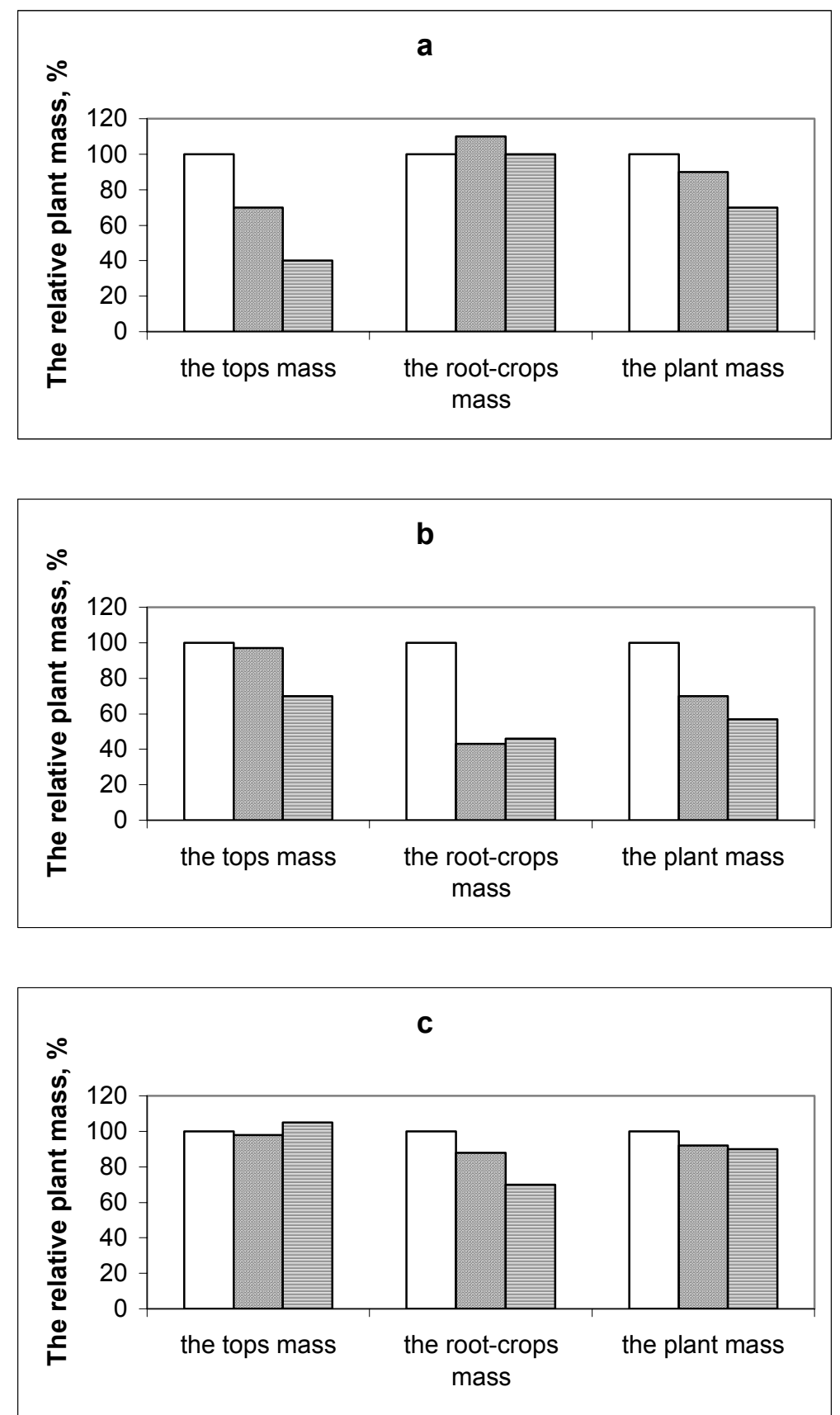

Fig. 3. The relative dry mass of the beet (a), carrot (b) and radish (c), grown on the SLS in the conveyor mode with the introduction of the inedible plant biomass into the substrate (the mass of the plants grown on the newly-prepared SLS - generation 1 is taken as $100 \%$ ) 
the inedible biomass of the studied plants into the SLS. The causes of the productivity drop may be connected with introducing great amounts of the organic substance and the microorganisms growth connected with it. These may also be connected with the compounds present in the inedible biomass which are unable to inhibit the plant growth.

The experiments on studying the influence of introducing dry straw into the SLS for the subsequent growing on it wheat and radish, also showed that this straw introduction leads to decreasing the plant productivity, which is evidently connected with not so much the quantity of the introduced organic substance, but with the peculiarities of its composition.

Among the methods of removing the negative influence of introducing the vegetable waste studied in the work, the most effective one turned out to be the mineralization applied by the method of the "wet incineration" with hydrogen peroxide developed by Yu. A. Kudenko. The application of this method of utilizing the inedible biomass alongside with growing the plants on the SLS is hardly expedient as it will lead to the substrate degradation.

As it was already noted, in the earlier experiments conducted on the experimental modeling of closed ecological systems (Gros et al., 2003), when growing radish and wheat with a common irrigation solution, there were obtained the productivities of these plants close to their productivity when grown on expanded clay aggregate (radish) and using a subirrigation method (wheat) with the utilization of standard mineral solutions. But the productivity of the wheat grains $(1.1-1.2 \mathrm{~kg} / \mathrm{m} 2)$ was more than 2 times lower than the productivity of the wheat grains grown on the newly - prepared SLS (Zolotukhin et al., 2005). Thus, even a multi-stage preparation of the straw before introducing it into the SLS, does not completely solve the problem of inhibiting the plant growth processes connected with introducing straw into the substrate.

Taking into account that the utilization of the inedible plant biomass in the SLS is connected with oxygen consumption and the release of carbon dioxide, to stabilize the composition of the atmosphere in the BLSS, it is necessary to increase the production of the edible photosynthetic mass (as compared to the production of the edible biomass when using neutral substrates for growing plants). It is desirable that the sown area should not be increased. That is why the increase in the productivity of the biocenosis edible biomass is a very important task when including the SLS in the BLSS. Solving this problem will allow to recommend using the SLS as a substrate for growing plants and thus increasing the degree of closing the mass exchange processes in the BLSS without considerable increase in energy consumption for the photosynthetic activity of vegetable biocenosis.

The work is supported by the project 5.16 of the Siberian Branch of the Russian Academy of Sciences.

\section{References}

Gitelson I.I., Lisovsky G.M., Mac Elroy R.D. (2003) Man-Made Closed Ecological Systems. Taylor and Francis, London, 402 p.

Gros J.B., Lasseur Ch., Tikhomirov A.A. Manukovsky N.S., Ushakova S.A, Zolotukhin I.G,. Gribovskaya I.V, Kovalev V.S (2003) Soil- Like Substrate for Plant Growing Derived from Inedible Plant Mass: Preparing, Composition, Fertility. Acta Horticulture (ISHS) 644: 151-155.

Kudenko Yu.A., Gribovskaya I.A., Pavlenko R.A. (1997) Mineralization of Wastes of Human Vital Activity and Plants in LSS. Acta Astronautica 41: 193-196. 
Kudenko Yu.A., Gribovskaya I.A., Zolotukhin I.G. (2000) Physical-Chemical Treatment of Waste: a Way to Close Turmover of Elements in LSS. Acta Astronautica 46: 585-589.

Lisovsky G.M., Shilenko M.P. (1979) Choosing the Structure and Conditions of Forming Higher Plant Units // Lisovsky G.M. (ed.) The Closed System "Man - Higher Plants". Nauka, Novosibirsk, p. 38-52.

Manukovsky N.S., Kovalev V.S., Rygalov V.Ye., Zolotukhin I.G. (1997) Waste Bioregeneration in Life Support Ces: Development of Soil Organic Substrate. Adv. Space Res. 20: 1827-1832.

Tikhomirov A.A., Ushakova S.A., Manukovsky N.S., Lisovsky G.M., Kudenko Yu.A., Kovalev V.S., Gubanov V.G., Barkhatov Yu.V., Gribovskaya I.V., Zolotukhin I.G., Gros J.B., Lasseu Ch. (2003a) Mass Exchange in an Experimental New-Generation Life Support System Model Based on Biological Regeneration of Environment Adv. Space Res. 31: 1711-1720.

Tikhomirov A.A., Ushakova S.A., Manukovsky N.S. Lisovsky G.M., Kudenko Yu.A., Kovalev V.S., Gribovskaya I.V., Tirranen L.S., Zolotukhin I.G. (2003b) Synthesis of Biological and Utilization of Plants Wastes in a Physical Model of Biological Life-Support System. Acta Astronautica 53: 249257.

Zolotukhin I.G., Tikhomirov A.A., Kudenko Yu.A., Gribovskaya I.A. (2005) Biological and Physicochemical Methods for Utilization of Plant Wastes and Human Exometabolites for Increasing Internal Cycling and Closure of Life Support Systems. Adv. Space Res. 35: 1559-1562. 